TAUP-2115-93

\title{
Equilibrium Relativistic Mass Distribution for Indistinguishable Events
}

\author{
L. Burakovsky* and L.P. Horwitz \\ School of Physics and Astronomy \\ Raymond and Beverly Sackler Faculty of Exact Sciences \\ Tel-Aviv University, Tel-Aviv 69978, ISRAEL
}

\begin{abstract}
A manifestly covariant relativistic statistical mechanics of the system of $N$ indistinguishable events with motion in space-time parametrized by an invariant "historical time" $\tau$ is considered. The relativistic mass distribution for such a system is obtained from the equilibrium solution of the generalized relativistic Boltzmann equation by integration over angular and hyperbolic angular variables. All the characteristic averages are calculated. Expressions for the pressure and the density of events are found and the relativistic equation of state is obtained. The Galilean limit is considered; the theory is shown to pass over to the usual nonrelativistic statistical mechanics of indistinguishable particles.
\end{abstract}

Key words: special relativity, relativistic Bose-Einstein/Fermi-Dirac, mass distribution

PACS: 03.30.+p, 05.20.Gg, 05.30.Ch, 98.20.-d

\footnotetext{
*Bitnet:BURAKOV@TAUNIVM.

${ }^{\dagger}$ Bitnet:HORWITZ@TAUNIVM. Also at Department of Physics, Bar-Ilan University, RamatGan, Israel
} 


\section{Introduction}

With this paper we continue a series of works on manifestly covariant relativistic statistical mechanics. In a previous paper [1] a manifestly covariant generalized relativistic Boltzmann equation for both cases of the system of $N$ distinguishable and indistinguishable events treated simultaneously has been made in the framework of a manifestly covariant classical and quantum mechanics [2]. These events, considered as the fundamental dynamical objects of the theory, move in an $8 N$-dimensional phase space. Their motion is parametrized by a continuous Poincaré invariant parameter $\tau$ called the "historical time". The collection of events (called "concatenation" [3]) along each world line corresponds to a particle in the usual sense; e.g., the Maxwell conserved current is an integral over the history of the charged event [4]. Hence the evolution of the state of the $N$-event system describes the history in space and time of an $N$-particle system.

A generalized relativistic Boltzmann equation was used to prove the $H$-theorem for evolution in $\tau$. In the equilibrium limit the covariant forms of the known nonrelativistic statistical distributions were obtained. Since these distributions are the distributions of the 4-momenta of the events, $m^{2}=-p^{2}=-p^{\mu} p_{\mu}$ is a random variable in a relativistic ensemble.

The case of distinguishable events was treated in a series of papers [5], [6], [7]. In

[5] the simplest case of a narrow mass shell $p^{2}=-m^{2} \cong-M^{2}$ was considered, where $M$ is a given fixed parameter with the dimension of mass (an intrinsic property of the particles), assumed to be the same for all the particles of the system. The results obtained in this approximation are in agreement with the well-known results of Synge 8] from an on-mass-shell relativistic kinetic theory.

In [6] the general case $0 \leq m<\infty$ was considered and a relativistic mass distribution was obtained. It has been shown [7] that in the Galilean limit the statistical mechanics of distinguishable events considered in [6] passes over to the usual nonrelativistic statistical mechanics of distinguishable particles.

In the present paper we consider the case of indistinguishable events. We obtain a relativistic mass distribution for this case, from the equilibrium solution of the manifestly covariant generalized Boltzmann equation obtained in [1], by integration over angular and hyperbolic angular variables. We calculate all the characteristic averages, obtain expressions for the energy-momentum tensor, pressure and density of the events, and derive the relativistic equation of state.

We show that in the Galilean limit the theory which we discuss here passes over to the usual nonrelativistic statistical mechanics of indistinguishable particles.

Finally, the criteria of validity of the theory presented in this paper are obtained. 


\section{Relativistic mass distribution}

We begin with the equilibrium relativistic distribution function obtained in [1] (we use the metric $g^{\mu \nu}=(-,+,+,+)$ and $\left.q \equiv q^{\mu}, p \equiv p^{\mu}\right)$,

$$
f_{0}(q, p)=C(q) \frac{1}{e^{-A(q)\left(p+p_{c}\right)^{2}-B(q)} \mp 1} \equiv C(q) \frac{e^{A(q)\left(p+p_{c}\right)^{2}+B(q)}}{1 \mp e^{A(q)\left(p+p_{c}\right)^{2}+B(q)}}, \quad A(q)>0 .
$$

The function (1) must be normalized, according to [1], as

$$
n(q)=\int f_{0}(q, p) d^{4} p=C(q) \int d^{4} p \frac{e^{A\left(p+p_{c}\right)^{2}+B}}{1 \mp e^{A\left(p+p_{c}\right)^{2}+B}}
$$

where $n(q)$ is the total number of events per unit space-time volume in the system in the neighborhood of the point $q$. By expanding the denominator in the integrand into a power series and introducing hyperbolic variables

$$
\Omega^{4}: m \geq 0,0 \leq \theta \leq \pi, 0 \leq \varphi<2 \pi,-\infty<\beta<\infty
$$

we can rewrite (2) as follows:

$$
\begin{aligned}
n(q)= & C(q) \sum_{n=1}^{\infty}( \pm 1)^{n+1} \int_{\Omega^{4}} d^{4} p e^{n A\left(p+p_{c}\right)^{2}+n B} \\
= & C(q) \sum_{n=1}^{\infty}( \pm 1)^{n+1} \int_{\Omega^{4}} m^{3} \sinh ^{2} \beta \sin \theta d m d \beta d \theta d \varphi \\
& \times e^{-n A m^{2}-2 n A m_{c} m \cosh \beta-n\left(A m_{c}^{2}-B\right)} .
\end{aligned}
$$

After some calculations [9] we obtain the normalization relation

$$
n(q)=C(q) \frac{\pi}{2 A^{2}} \sum_{n=1}^{\infty} \frac{( \pm 1)^{n+1}}{n^{2}} e^{-n\left(A m_{c}^{2}-B\right)} \Psi\left(2,2 ; A m_{c}^{2}\right) .
$$

Here $\Psi(a, b ; z)$ is the confluent hypergeometrical function of $z$ (ref.[10], p.257, sec.6.6). After integration over angular and hyperbolic angular variables we obtain from (3) the expression

$$
n(q)=C(q) \frac{2 \pi}{A m_{c}} \sum_{n=1}^{\infty} \frac{( \pm 1)^{n+1}}{n} \int_{0}^{\infty} d m m^{2} e^{-n\left(A m_{c}^{2}-B\right)} e^{-n A m^{2}} K_{1}\left(2 n A m_{c} m\right),
$$

from which we identify the mass distribution function

$$
f(m)=D \sum_{n=1}^{\infty} \frac{( \pm 1)^{n+1}}{n} m^{2} e^{-n\left(A m_{c}^{2}-B\right)} e^{-n A m^{2}} K_{1}\left(2 n A m_{c} m\right)
$$


where

$$
D^{-1}=\frac{m_{c}}{4 A} \sum_{n=1}^{\infty} \frac{( \pm 1)^{n+1}}{n^{2}} e^{-n\left(A m_{c}^{2}-B\right)} \Psi\left(2,2 ; n A m_{c}^{2}\right)
$$

so that $f(m)$ is normalized according to

$$
\int_{0}^{\infty} f(m) d m=1
$$

In (5) and (6) $K_{1}$ is the Bessel function of the third kind (imaginary argument), where, in general,

$$
K_{\nu}(z)=\frac{\pi i}{2} e^{\pi i \nu / 2} H_{\nu}^{(1)}(i z) .
$$

With the help of this distribution one can obtain the local average value of an arbitrary function of mass $\phi(m)$ :

$$
\langle\phi(m)\rangle_{q}=\int_{0}^{\infty} \phi(m) f(m) d m .
$$

Let us obtain, for example, the local average values of mass and mass squared in relativistic gas which represent the first two moments of the distribution (6) (factors $m^{\ell}$ result in a change in the first argument of the confluent hypergeometric function and its normalization):

$$
\begin{gathered}
\langle m\rangle_{q}=\frac{3 \pi}{8 A^{\frac{1}{2}}} \frac{\sum_{n=1}^{\infty}\left\{( \pm 1)^{n+1} e^{-n\left(A m_{c}^{2}-B\right)} \Psi\left(\frac{5}{2}, 2 ; n A m_{c}^{2}\right) / n^{\frac{5}{2}}\right\}}{\sum_{n=1}^{\infty}\left\{( \pm 1)^{n+1} e^{-n\left(A m_{c}^{2}-B\right)} \Psi\left(2,2 ; n A m_{c}^{2}\right) / n^{2}\right\}} \\
\left\langle m^{2}\right\rangle_{q}=\frac{2}{A} \frac{\sum_{n=1}^{\infty}\left\{( \pm 1)^{n+1} e^{-n\left(A m_{c}^{2}-B\right)} \Psi\left(3,2 ; n A m_{c}^{2}\right) / n^{3}\right\}}{\sum_{n=1}^{\infty}\left\{( \pm 1)^{n+1} e^{-n\left(A m_{c}^{2}-B\right)} \Psi\left(2,2 ; n A m_{c}^{2}\right) / n^{2}\right\}}
\end{gathered}
$$

More generally,

$$
\left\langle m^{\ell}\right\rangle_{q}=\Gamma\left(\frac{\ell}{2}+1\right) \Gamma\left(\frac{\ell}{2}+2\right) A^{-\frac{\ell}{2}} \frac{\sum_{n=1}^{\infty}\left\{( \pm 1)^{n+1} e^{-n\left(A m_{c}^{2}-B\right)} \Psi\left(2+\frac{\ell}{2}, 2 ; n A m_{c}^{2}\right) / n^{2+\frac{\ell}{2}}\right\}}{\sum_{n=1}^{\infty}\left\{( \pm 1)^{n+1} e^{-n\left(A m_{c}^{2}-B\right)} \Psi\left(2,2 ; n A m_{c}^{2}\right) / n^{2}\right\}} .
$$

Now, as in [5],[6], we define absolute temperature through the relation

$$
2 A m_{c}=\frac{1}{k_{B} T}
$$

which implies that in thermal equilibrium $A m_{c}$ is independent of $q$. Hence

$$
\langle m\rangle=\frac{3 \pi}{8} \sqrt{2 m_{c} k_{B} T} \frac{\sum_{n=1}^{\infty}\left\{( \pm 1)^{n+1} e^{-n\left(\frac{m_{c}}{2 k_{B} T}-B\right)} \Psi\left(\frac{5}{2}, 2 ; n \frac{m_{c}}{2 k_{B} T}\right) / n^{\frac{5}{2}}\right\}}{\sum_{n=1}^{\infty}\left\{( \pm 1)^{n+1} e^{-n\left(\frac{m_{c}}{2 k_{B} T}-B\right)} \Psi\left(2,2 ; n \frac{m_{c}}{2 k_{B} T}\right) / n^{2}\right\}} .
$$

In the limit $T \rightarrow 0$ it follows from the asymptotic formula for $z \rightarrow \infty$ [10

$\Psi(a, b ; z) \sim z^{-a}\left[1+\sum_{n=1}^{\infty}(-1)^{n} \frac{a(a+1) \cdots(a+n-1)(1+a-b)(2+a-b) \cdots(n+a-b)}{n ! z^{n}}\right]$ 
that

$$
\langle m\rangle \cong \frac{3 \pi}{4} k_{B} T \frac{\sum_{n=1}^{\infty}\left\{( \pm 1)^{n+1} e^{-n\left(\frac{m_{c}}{2 k_{B} T}-B\right)} / n^{5}\right\}}{\sum_{n=1}^{\infty}\left\{( \pm 1)^{n+1} e^{-n\left(\frac{m_{c}}{2 k_{B} T}-B\right)} / n^{4}\right\}} .
$$

One can also obtain in this limit

$$
\left\langle m^{2}\right\rangle \cong 8\left(k_{B} T\right)^{2} \frac{\sum_{n=1}^{\infty}\left\{( \pm 1)^{n+1} e^{-n\left(\frac{m_{c}}{2 k_{B} T}-B\right)} / n^{6}\right\}}{\sum_{n=1}^{\infty}\left\{( \pm 1)^{n+1} e^{-n\left(\frac{m_{c}}{2 k_{B} T}-B\right)} / n^{4}\right\}} .
$$

Let us now calculate the first two moments of the distribution (1):

$$
\begin{gathered}
\left\langle p^{\mu}\right\rangle_{q}=\frac{\int d^{4} p p^{\mu} f_{0}(q, p)}{\int d^{4} p f_{0}(q, p)}=\frac{\int d^{4} p p^{\mu} \frac{e^{A\left(p+p_{c}\right)^{2}+B}}{1 \mp e^{A\left(p+p_{c}\right)^{2}+B}}}{\int d^{4} p \frac{e^{A\left(p+p_{c}\right)^{2}+B}}{1 \mp e^{A\left(p+p_{c}\right)^{2}+B}}}=\frac{\sum_{n=1}^{\infty}( \pm 1)^{n+1} \int d^{4} p p^{\mu} e^{n A\left(p+p_{c}\right)^{2}+n B}}{\sum_{n=1}^{\infty}( \pm 1)^{n+1} \int d^{4} p e^{n A\left(p+p_{c}\right)^{2}+n B}} \\
\left\langle p^{\mu} p^{\nu}\right\rangle_{q}=\frac{\int d^{4} p p^{\mu} p^{\nu} f_{0}(q, p)}{\int d^{4} p f_{0}(q, p)}=\frac{\sum_{n=1}^{\infty}( \pm 1)^{n+1} \int d^{4} p p^{\mu} p^{\nu} e^{n A\left(p+p_{c}\right)^{2}+n B}}{\sum_{n=1}^{\infty}( \pm 1)^{n+1} \int d^{4} p e^{n A\left(p+p_{c}\right)^{2}+n B}}
\end{gathered}
$$

Define the following functions:

$$
\begin{aligned}
& F_{0}=\sum_{n=1}^{\infty}( \pm 1)^{n+1} \int d^{4} p e^{n A\left(p+p_{c}\right)^{2}+n B} \equiv \int d^{4} p f_{0}(q, p) \\
& F_{1}=\sum_{n=1}^{\infty} \frac{( \pm 1)^{n+1}}{n} \int d^{4} p e^{n A\left(p+p_{c}\right)^{2}+n B} \\
& F_{2}=\sum_{n=1}^{\infty} \frac{( \pm 1)^{n+1}}{n^{2}} \int d^{4} p e^{n A\left(p+p_{c}\right)^{2}+n B}
\end{aligned}
$$

We have

$$
\begin{aligned}
\left\langle p^{\mu}\right\rangle_{q} & =F_{0}^{-1}\left(\frac{1}{2 A} \frac{\partial F_{1}}{\partial p_{c \mu}}-p_{c}^{\mu}\right) \\
\left\langle p^{\mu} p^{\nu}\right\rangle_{q} & =F_{0}^{-1}\left(\frac{1}{4 A^{2}} \frac{\partial^{2} F_{2}}{\partial p_{c \mu} \partial p_{c \nu}}-\left\langle p^{\mu}\right\rangle p_{c}^{\nu}-\left\langle p^{\nu}\right\rangle p_{c}^{\mu}-p_{c}^{\mu} p_{c}^{\nu}\right)
\end{aligned}
$$

Calculating $F_{1}$ and $F_{2}$ in explicit form and using the relations

$$
\begin{aligned}
\frac{\partial}{\partial p_{c \mu}} & =-2 p_{c}^{\mu} \frac{\partial}{\partial m_{c}^{2}} \\
\frac{\partial^{2}}{\partial p_{c \mu} \partial p_{c \nu}} & =4 p_{c}^{\mu} p_{c}^{\nu} \frac{\partial^{2}}{\partial\left(m_{c}^{2}\right)^{2}}-2 g^{\mu \nu} \frac{\partial}{\partial m_{c}^{2}}
\end{aligned}
$$

we obtain with the help of the formula (ref.[10], p.257, sec.6.6)

$$
\frac{d}{d z} \Psi(a, b ; z)=-a \Psi(a+1, b+1 ; z)
$$




$$
\begin{aligned}
\left\langle p^{\mu}\right\rangle_{q}= & 2 p_{c}^{\mu} \frac{\sum_{n=1}^{\infty}\left\{( \pm 1)^{n+1} e^{-n\left(A m_{c}^{2}-B\right)} \Psi\left(3,3 ; n A m_{c}^{2}\right) / n^{2}\right\}}{\sum_{n=1}^{\infty}\left\{( \pm 1)^{n+1} e^{-n\left(A m_{c}^{2}-B\right)} \Psi\left(2,2 ; n A m_{c}^{2}\right) / n^{2}\right\}} \\
\left\langle p^{\mu} p^{\nu}\right\rangle_{q}= & 6 p_{c}^{\mu} p_{c}^{\nu} \frac{\sum_{n=1}^{\infty}\left\{( \pm 1)^{n+1} e^{-n\left(A m_{c}^{2}-B\right)} \Psi\left(4,4 ; n A m_{c}^{2}\right) / n^{2}\right\}}{\sum_{n=1}^{\infty}\left\{( \pm 1)^{n+1} e^{-n\left(A m_{c}^{2}-B\right)} \Psi\left(2,2 ; n A m_{c}^{2}\right) / n^{2}\right\}} \\
& +\frac{g^{\mu \nu}}{A} \frac{\sum_{n=1}^{\infty}\left\{( \pm 1)^{n+1} e^{-n\left(A m_{c}^{2}-B\right)} \Psi\left(3,3 ; n A m_{c}^{2}\right) / n^{3}\right\}}{\sum_{n=1}^{\infty}\left\{( \pm 1)^{n+1} e^{-n\left(A m_{c}^{2}-B\right)} \Psi\left(2,2 ; n A m_{c}^{2}\right) / n^{2}\right\}}
\end{aligned}
$$

As in [5],[6], we make a Lorentz transformation to the local average motion rest frame moving with the relative velocity

$$
\mathbf{u}=\frac{\mathbf{p}_{c}}{E_{c}}
$$

in order to obtain the local energy density.

The rest frame energy is

$$
\left\langle E^{\prime}\right\rangle_{q}=\frac{\langle E\rangle_{q}-\mathbf{u} \cdot \mathbf{p}}{\sqrt{1-\mathbf{u}^{2}}}
$$

so that

$$
\left\langle E^{\prime}\right\rangle_{q}=2 m_{c} \frac{\sum_{n=1}^{\infty}\left\{( \pm 1)^{n+1} e^{-n\left(A m_{c}^{2}-B\right)} \Psi\left(3,3 ; n A m_{c}^{2}\right) / n^{2}\right\}}{\sum_{n=1}^{\infty}\left\{( \pm 1)^{n+1} e^{-n\left(A m_{c}^{2}-B\right)} \Psi\left(2,2 ; n A m_{c}^{2}\right) / n^{2}\right\}} .
$$

To obtain the pressure and the density of events in our ensemble, as in [5],[6], we study the particle energy-momentum tensor defined by the $R^{4}$ density

$$
T^{\mu \nu}(q)=\sum_{i} \int d \tau \frac{p_{i}^{\mu} p_{i}^{\nu}}{M} \delta^{4}\left(q-q_{i}(\tau)\right)
$$

Using the result of [5]

$$
\left\langle T^{\mu \nu}(q)\right\rangle_{q}=T_{\triangle V} \int d^{4} p f_{0}(q, p) \frac{p^{\mu} p^{\nu}}{M}
$$

and the expression (26) for $\left\langle p^{\mu} p^{\nu}\right\rangle_{q}$, we obtain

$$
\begin{aligned}
\left\langle T^{\mu \nu}(q)\right\rangle_{q} & =\frac{T_{\triangle V} n(q)}{M}\left[\frac{g^{\mu \nu}}{A} \frac{\sum_{n=1}^{\infty}\left\{( \pm 1)^{n+1} e^{-n\left(A m_{c}^{2}-B\right)} \Psi\left(3,3 ; n A m_{c}^{2}\right) / n^{3}\right\}}{\sum_{n=1}^{\infty}\left\{( \pm 1)^{n+1} e^{-n\left(A m_{c}^{2}-B\right)} \Psi\left(2,2 ; n A m_{c}^{2}\right) / n^{2}\right\}}\right. \\
& \left.+6 p_{c}^{\mu} p_{c}^{\nu} \frac{\sum_{n=1}^{\infty}\left\{( \pm 1)^{n+1} e^{-n\left(A m_{c}^{2}-B\right)} \Psi\left(4,4 ; n A m_{c}^{2}\right) / n^{2}\right\}}{\sum_{n=1}^{\infty}\left\{( \pm 1)^{n+1} e^{-n\left(A m_{c}^{2}-B\right)} \Psi\left(2,2 ; n A m_{c}^{2}\right) / n^{2}\right\}}\right] .
\end{aligned}
$$

In this expression $T_{\triangle V}$ is the average passage interval in $\tau$ for the events which pass through the small four-volume $\triangle V$ over the point $q$ of $R^{4}$.

The formula for the stress-energy tensor of a perfect fluid has the form $[5,(3.39)]$

$$
\left\langle T^{\mu \nu}(q)\right\rangle_{q}=p g^{\mu \nu}-(p+\rho) \frac{\left\langle p^{\mu}\right\rangle_{q}\left\langle p^{\nu}\right\rangle_{q}}{\left\langle p^{\lambda}\right\rangle_{q}\left\langle p_{\lambda}\right\rangle_{q}}
$$


where $p$ is the pressure and $\rho$ is the density of energy at $q$.

According to (25),

$$
\frac{\left\langle p^{\mu}\right\rangle_{q}}{\sqrt{-\left\langle p^{\lambda}\right\rangle_{q}\left\langle p_{\lambda}\right\rangle_{q}}}=\frac{p_{c}^{\mu}}{m_{c}}
$$

hence

$$
p=\frac{T_{\triangle V} n(q)}{A M} \frac{\sum_{n=1}^{\infty}\left\{( \pm 1)^{n+1} e^{-n\left(A m_{c}^{2}-B\right)} \Psi\left(3,3 ; n A m_{c}^{2}\right) / n^{3}\right\}}{\sum_{n=1}^{\infty}\left\{( \pm 1)^{n+1} e^{-n\left(A m_{c}^{2}-B\right)} \Psi\left(2,2 ; n A m_{c}^{2}\right) / n^{2}\right\}} .
$$

and

$$
p+\rho=\frac{6 T_{\triangle V} n(q) m_{c}^{2}}{M} \frac{\sum_{n=1}^{\infty}\left\{( \pm 1)^{n+1} e^{-n\left(A m_{c}^{2}-B\right)} \Psi\left(4,4 ; n A m_{c}^{2}\right) / n^{2}\right\}}{\sum_{n=1}^{\infty}\left\{( \pm 1)^{n+1} e^{-n\left(A m_{c}^{2}-B\right)} \Psi\left(2,2 ; n A m_{c}^{2}\right) / n^{2}\right\}} .
$$

To interpret these results, as in [5],[6], we should calculate the average (conserved) particle four-current, which has the microscopic form

$$
J^{\mu}(q)=\sum_{i} \int \frac{p_{i}^{\mu}}{M} \delta^{4}\left(q-q_{i}(\tau)\right) d \tau .
$$

Using the result of $[5]$

$$
\left\langle J^{\mu}(q)\right\rangle_{q}=T_{\triangle V} \int d^{4} p \frac{p^{\mu}}{M} f_{0}(q, p)
$$

and expression (25) for $\left\langle p^{\mu}\right\rangle_{q}$, we obtain

$$
\left\langle J^{\mu}(q)\right\rangle_{q}=\frac{2 T_{\triangle V} n(q)}{M} p_{c}^{\mu} \frac{\sum_{n=1}^{\infty}\left\{( \pm 1)^{n+1} e^{-n\left(A m_{c}^{2}-B\right)} \Psi\left(3,3 ; n A m_{c}^{2}\right) / n^{2}\right\}}{\sum_{n=1}^{\infty}\left\{( \pm 1)^{n+1} e^{-n\left(A m_{c}^{2}-B\right)} \Psi\left(2,2 ; n A m_{c}^{2}\right) / n^{2}\right\}} .
$$

In the local rest frame $p_{c}^{\mu}=\left(m_{c}, \mathbf{0}\right)$,

$$
\left\langle J^{0}(q)\right\rangle_{q}=\frac{2 T_{\triangle V} n(q) m_{c}}{M} \frac{\sum_{n=1}^{\infty}\left\{( \pm 1)^{n+1} e^{-n\left(A m_{c}^{2}-B\right)} \Psi\left(3,3 ; n A m_{c}^{2}\right) / n^{2}\right\}}{\sum_{n=1}^{\infty}\left\{( \pm 1)^{n+1} e^{-n\left(A m_{c}^{2}-B\right)} \Psi\left(2,2 ; n A m_{c}^{2}\right) / n^{2}\right\}} .
$$

Defining the density of paricles per unit space volume as

$$
N_{0}(q)=\left\langle J^{0}(q)\right\rangle_{q},
$$

we obtain the relativistic equation of state

$$
\begin{aligned}
& p=\frac{N_{0}}{2 A m_{c}} \frac{\sum_{n=1}^{\infty}\left\{( \pm 1)^{n+1} e^{-n\left(A m_{c}^{2}-B\right)} \Psi\left(3,3 ; n A m_{c}^{2}\right) / n^{3}\right\}}{\sum_{n=1}^{\infty}\left\{( \pm 1)^{n+1} e^{-n\left(A m_{c}^{2}-B\right)} \Psi\left(3,3 ; n A m_{c}^{2}\right) / n^{2}\right\}} \\
& =N_{0} k_{B} T \frac{\sum_{n=1}^{\infty}\left\{( \pm 1)^{n+1} e^{-n\left(\frac{m_{c}}{2 k_{B} T}-B\right)} \Psi\left(3,3 ; n \frac{m_{c}}{2 k_{B} T}\right) / n^{3}\right\}}{\sum_{n=1}^{\infty}\left\{( \pm 1)^{n+1} e^{-n\left(\frac{m_{c}}{2 k_{B} T}-B\right)} \Psi\left(3,3 ; n \frac{m_{c}}{2 k_{B} T}\right) / n^{2}\right\}} .
\end{aligned}
$$

In limiting case $T \rightarrow 0$ the general expression for the distribution function (6) can be simplified. Indeed, it is seen from (17) and (27) that in this case $\left\langle m^{2}\right\rangle \sim\left(k_{B} T\right)^{2} \ll$ 
$\left\langle E^{\prime}\right\rangle m_{c} \sim m_{c}\left(k_{B} T\right)$. Therefore, one can neglect $m^{2}=-p^{2}$ in comparison with $2 A p p_{c}$ in the exponent of the initial distribution (1); hence, we begin with

$$
f_{0}^{\prime}(p, q) \cong C(q) \frac{1}{e^{A m_{c}^{2}-2 A p p_{c}-B} \mp 1},
$$

and after integration and normalization obtain

$$
\begin{gathered}
f^{0}(m)=D^{0} \sum_{n=1}^{\infty} \frac{( \pm 1)^{n+1}}{n} m^{2} e^{-n\left(A m_{c}^{2}-B\right)} K_{1}\left(2 n A m_{c} m\right) \\
\left(D^{0}\right)^{-1}=\frac{2}{\left(2 A m_{c}\right)^{3}} \sum_{n=1}^{\infty} \frac{( \pm 1)^{n+1}}{n^{4}} e^{-n\left(A m_{c}^{2}-B\right)}
\end{gathered}
$$

The distribution function (42) gives

$$
\begin{gathered}
\left\langle m^{\ell}\right\rangle^{0}=\Gamma\left(\frac{\ell}{2}+1\right) \Gamma\left(\frac{\ell}{2}+2\right)\left(A m_{c}\right)^{-\ell} \frac{\sum_{n=1}^{\infty}\left\{( \pm 1)^{n+1} e^{-n\left(A m_{c}^{2}-B\right)} / n^{4+\ell}\right\}}{\sum_{n=1}^{\infty}\left\{( \pm 1)^{n+1} e^{-n\left(A m_{c}^{2}-B\right)} / n^{4}\right\}} \\
=\Gamma\left(\frac{\ell}{2}+1\right) \Gamma\left(\frac{\ell}{2}+2\right)\left(2 k_{B} T\right)^{\ell} \frac{\sum_{n=1}^{\infty}\left\{( \pm 1)^{n+1} e^{-n\left(\frac{m_{c}}{2 k_{B} T}-B\right)} / n^{4+\ell}\right\}}{\sum_{n=1}^{\infty}\left\{( \pm 1)^{n+1} e^{-n\left(\frac{m_{c}}{2 k_{B} T}-B\right)} / n^{4}\right\}}
\end{gathered}
$$

which coincides with the low-temperature limit of (12). Similarly, one can obtain in this case

$$
\left\langle p^{\mu}\right\rangle^{0}=\frac{p_{c}^{\mu}}{m_{c}} 4 k_{B} T \frac{\sum_{n=1}^{\infty}\left\{( \pm 1)^{n+1} e^{-n\left(\frac{m_{c}}{2 k_{B} T}-B\right)} / n^{5}\right\}}{\sum_{n=1}^{\infty}\left\{( \pm 1)^{n+1} e^{-n\left(\frac{m_{c}}{2 k_{B} T}-B\right)} / n^{4}\right\}},
$$

which coincides with the low-temperature limit of (25).

To interpret these results, consider the initial equilibrium distribution function $f_{0}(q, p)$ in the local rest frame $p_{c}^{\mu}=\left(m_{c}, \mathbf{0}\right)$. Using (13) one obtains

$$
\begin{aligned}
f_{0}(q, p) & =C(q) \frac{1}{e^{-A\left(p+p_{c}\right)^{2}-B} \mp 1}=C(q) \frac{1}{e^{A m^{2}+2 A m_{c} E+\left(A m_{c}^{2}-B\right)} \mp 1} \\
& =C(q) \frac{1}{\exp \left\{\frac{1}{k_{B} T}\left[E+\frac{m^{2}}{2 m_{c}}+\left(\frac{m_{c}}{2}-k_{B} T \cdot B\right)\right]\right\} \mp 1} .
\end{aligned}
$$

Therefore, one can put

$$
\begin{gathered}
m_{c} \equiv \frac{M}{\mu_{K}} \\
B \equiv \frac{1}{k_{B} T}\left(\mu+\frac{m_{c}}{2}\right),
\end{gathered}
$$

and hence to identify (46) with the grand canonical distribution function

$$
\frac{1}{\exp \left\{\frac{1}{k_{B} T}\left[E-\mu+\mu_{K} \frac{m^{2}}{2 M}\right]\right\} \mp 1},
$$


obtained previously in [11] for the static Gibbs ensembles. In the latter formula $\mu$ and $\mu_{K}$ are relativistic chemical and mass potentials, respectively [11]. Hence $A m_{c}^{2}-B \equiv-\mu / k_{B} T$, and $\exp \left\{-n\left(A m_{c}^{2}-B\right)\right\}$ should be identified with $\exp \left(\frac{n \mu}{k_{B} T}\right)$ in all formulas containing this exponent, throughout the paper.

For example, the relativistic equation of state (40) now reads

$$
p=N_{0} k_{B} T \frac{\sum_{n=1}^{\infty}\left\{( \pm 1)^{n+1} e^{\frac{n \mu}{k_{B} T}} \Psi\left(3,3 ; \frac{n M}{2 \mu_{K} k_{B} T}\right) / n^{3}\right\}}{\sum_{n=1}^{\infty}\left\{( \pm 1)^{n+1} e^{\frac{n \mu}{k_{B} T}} \Psi\left(3,3 ; \frac{n M}{2 \mu_{K} k_{B} T}\right) / n^{2}\right\}} .
$$

Since at thermal equilibrium there is no dependence on $q$, according to [1], instead of $f_{0}(q, p)$ one can use equilibrium relativistic distribution function

$$
f_{0}(p)=\int d^{4} q f_{0}(q, p)=\frac{1}{e^{\left(E-\mu+\mu_{K} \frac{m^{2}}{2 M}\right) / k_{B} T} \mp 1},
$$

normalized as

$$
\int \frac{d^{4} p}{(2 \pi)^{4}} f_{0}(p)=n
$$

where $n \equiv \frac{N}{V^{(4)}}$ is the event number density.

\section{Galilean limit of relativistic mass distribution}

The Galilean limit of manifestly covariant relativistic theory which we discuss here has been considered in a series of papers [7],[11], [12], by taking $c \rightarrow \infty$ (compared to all other velocities). It has been shown [7] that in the Galilean limit the relativistic relation between the energy $E$ and the mass $m$

$$
E^{2}=m^{2}+\mathbf{p}^{2}
$$

transforms to

$$
E=m+\frac{\mathbf{p}^{2}}{2 M}
$$

where the Galilean mass $M$ coincides with the particle's intrinsic parameter. At the same time the quantity

$$
\eta=c^{2}(m-M)
$$

may take any value, however, finite, as $c \rightarrow \infty$; or, equivalently, $m=M\left(1+O\left(1 / c^{2}\right)\right)$.

In case of an equilibrium relativistic ensemble of distinguishable events such a transformation of the relativistic relation between $E$ and $m$ gives rise to the usual nonrelativistic Maxwell-Boltzmann distribution of $\mathbf{p}^{2} / 2 M$.

\footnotetext{
${ }^{1}$ Clearly, all the results of the paper, since obtained for thermal equilibrium, remain valid.
} 
Now we shall consider the case of indistinguishable events. We start with the low-temperature form (41) of initial equilibrium relativistic distribution (1) which is normalized as follows,

$$
n(q)=C(q) \int d^{4} p \frac{1}{e^{A m_{c}^{2}-2 A p p_{c}-B} \mp 1} .
$$

This integral written in the local rest frame takes the form

$$
\int d E d^{3} \mathbf{p} \sum_{n=1}^{\infty}( \pm 1)^{n+1} e^{-2 n A m_{c} E} e^{-n\left(A m_{c}^{2}-B\right)} \equiv \int d E d^{3} \mathbf{p} \sum_{n=1}^{\infty}( \pm 1)^{n+1} e^{-\frac{n E}{k_{B} T}} e^{\frac{n \mu}{k_{B} T}},
$$

or, in view of (53),(54) and the relation $-\triangle \leq \eta \leq \triangle[7]$,

$$
\sum_{n=1}^{\infty} \int_{M-\triangle}^{M+\triangle} d m e^{-\frac{n m}{k_{B} T}} \int d^{3} \mathbf{p} e^{-\frac{n}{k_{B} T}\left(\frac{\mathbf{p}^{2}}{2 M}-\mu\right)} .
$$

Integration on $m$ gives

$$
\int_{M-\triangle}^{M+\triangle} d m e^{-\frac{n m}{k_{B} T}}=2 \frac{k_{B} T}{n} e^{-\frac{n M}{k_{B} T}} \sinh \frac{n \triangle}{k_{B} T} .
$$

Since $\triangle$ may take any infinitesimal valuef, but not zero (it enters the normalization factor), we obtain in the limit $\triangle \rightarrow 0$

$$
\frac{k_{B} T}{n} \sinh \frac{n \triangle}{k_{B} T} \cong \triangle
$$

and therefore

$$
n(q)=2 \triangle C(q) \int d^{3} \mathbf{p} \sum_{n=1}^{\infty}( \pm 1)^{n+1} e^{-\frac{n}{k_{B} T}\left(\frac{\mathbf{p}^{2}}{2 M}-\mu+M\right)} \equiv 2 \triangle C(q) \int d^{3} \mathbf{p} \frac{1}{e^{\left.\frac{\mathbf{p}^{2}}{2 M}-\tilde{\mu}\right) / k_{B} T} \mp 1},
$$

which is the usual (normalized) nonrelativistic Bose-Einstein/Fermi-Dirac distribution for $e \equiv \frac{\mathbf{p}^{2}}{2 M}$,

$$
\begin{gathered}
f(e)=\tilde{D} \frac{e^{1 / 2}}{\exp \left(\frac{e-\tilde{\mu}}{k_{B} T}\right) \mp 1}, \\
\tilde{D}^{-1}=\Gamma\left(\frac{3}{2}\right)\left(k_{B} T\right)^{3 / 2} \sum_{n=1}^{\infty} \frac{( \pm 1)^{n+1}}{n^{3 / 2}} e^{\frac{n \tilde{\mu}}{k_{B} T}} .
\end{gathered}
$$

In the latter formulas

$$
\tilde{\mu}=\mu-M
$$

is the usual nonrelativistic chemical potential.

\footnotetext{
${ }^{2}$ It corresponds to infinitely sharp mass shell $\triangle=c^{2}|m-M|$ [7].
} 
A precise equation for $\mu=\mu\left(n, \mu_{K}, T\right)$ can be obtained from $(50),(51)$ by performing integration:

$$
n=\frac{1}{(2 \pi)^{3}} \frac{M^{2}}{\mu_{K}^{2}}\left(k_{B} T\right)^{2} \sum_{s=1}^{\infty} \frac{( \pm 1)^{s+1}}{s^{2}} e^{\frac{s \mu}{k_{B} T}} \Psi\left(2,2 ; \frac{s M}{2 \mu_{K} k_{B} T}\right) .
$$

The corresponding equation for the low-temperature case is obtained from $(41),(51)$, or directly from (63), using the asymptotic formula (15),

$$
n=\frac{\left(k_{B} T\right)^{4}}{2 \pi^{3}} \sum_{s=1}^{\infty} \frac{( \pm 1)^{s+1}}{s^{4}} e^{\frac{s \mu}{k_{B} T}} .
$$

By means of the formula

$$
\sum_{s=1}^{\infty} \frac{( \pm 1)^{s+1}}{s^{p}} e^{\frac{s \mu}{k_{B} T}} \equiv \sum_{s=1}^{\infty} \frac{( \pm 1)^{s+1}}{s^{p}} z^{s}= \pm \sum_{s=1}^{\infty} \frac{( \pm z)^{s}}{s^{p}}= \pm L i_{p}( \pm z), \quad z \equiv e^{\frac{\mu}{k_{B} T}},
$$

where

$$
L i_{\nu}(z) \equiv \sum_{k=1}^{\infty} \frac{z^{k}}{k^{\nu}}, \quad|z|<1 \quad \text { or } \quad|z|=1, \quad \text { Re } \nu>1,
$$

is the so-called polylogarithm [15], the equation (64) can be rewritten as follows,

$$
n= \pm \frac{\left(k_{B} T\right)^{4}}{2 \pi^{3}} L i_{4}\left( \pm e^{\frac{\mu}{k_{B} T}}\right)
$$

For comparison, the equation of nonrelativistic statistical mechanics for $\tilde{\mu}=\tilde{\mu}\left(N_{0}, T\right)$ is obtained from the normalization relation of the distribution function (59),

$$
\int \frac{d^{3} \mathbf{p}}{(2 \pi)^{3}} \frac{1}{e^{\left(\frac{\mathbf{p}^{2}}{2 M}-\tilde{\mu}\right) / k_{B} T} \mp 1}=N_{0}
$$

$N_{0}$ being defined by (39), and is found to be

$$
N_{0}= \pm\left(\frac{M k_{B} T}{2 \pi}\right)^{3 / 2} L i_{\frac{3}{2}}\left( \pm e^{\frac{\tilde{\mu}}{k_{B} T}}\right)
$$

\section{Validity criteria}

Now we wish to consider the limits of applicability of the theory which we discussed in the present paper and obtain explicit criteria for its validity.

\footnotetext{
${ }^{3}$ For $|z| \geq 1$ the function $L i_{\nu}(z)$ is defined as the analytic continuation of this series.
} 
It is clear from the general considerations that the theory is valid at medium and low temperatures still far from absolute zero, at which the degeneracy effects manifest themselves. The explicit criterions of its validity can be obtained as follows:

1) Since in any system of macroscoping size the spacing between successive momentum levels should be exceedingly small compared to the characteristic thermal momentum $k_{B} T$, one has f

$$
\frac{p_{n}^{\mu}-p_{n-1}^{\mu}}{k_{B} T}=\frac{\hbar}{L^{(\mu)} k_{B} T}<<1,
$$

where $L^{(\mu)}$ is a character size of a system in $\mu$-direction. Taking into account the four similar relations for $\mu=0,1,2,3,(70)$ can be rewritten as follows (in the system of units we use),

$$
\frac{N}{L^{4}\left(k_{B} T\right)^{4}}<<1, \quad \text { or } \frac{n}{\left(k_{B} T\right)^{4}}<<1,
$$

where $L$ is a character size of a system, $L^{4} \sim V^{(4)}$, the total four-volume, occupied by the system in four-dimensional space-time, and $n=\frac{N}{V^{(4)}}$ is the event number density. Hence, the criterion of validity is determined by a dimensionless number $\delta$, conventionally called the degeneracy parameter:

$$
\delta=\frac{n}{\left(k_{B} T\right)^{4}}<<1
$$

This criterion can be violated at low temperatures near absolute zero or at high event densities. The two possible situations constitute the so-called degenerate case and will be treated in the following research [13].

2) In order that the expansion

$$
\frac{1}{e^{-A\left(p+p_{c}\right)^{2}-B} \mp 1}=\sum_{n=1}^{\infty}( \pm 1)^{n+1} e^{n A\left(p+p_{c}\right)^{2}+n B}
$$

which is basic for the theory, be valid, we must have

$$
e^{A\left(p+p_{c}\right)^{2}+B}<1
$$

or, equivalently,

$$
-A\left(p+p_{c}\right)^{2}-B>0 .
$$

This relation can be rewritten in the local rest frame, using $\cosh \beta \geq 1$,

$$
A m^{2}+2 A m m_{c} \cosh \beta+A m_{c}^{2}-B \geq A\left(m+m_{c}\right)^{2}-B>0 .
$$

\footnotetext{
${ }^{4}$ At high temperatures it goes over to relativistic statistical mechanics of distinguishable events considered previously in [6].

${ }^{5}$ This condition implies that in the thermodynamic limit, summation over the discrete variables $n_{i}$ can be replaced by integration over the continuous variable $p$, where $n_{i}$ is the occupation number in the event energy-momentum space.
} 
Since the latter must be valid for any $m$, we deduce that

$$
B<A m_{c}^{2} \equiv \frac{m_{c}}{2 k_{B} T}
$$

Using the relation (48), $B \equiv \frac{m_{c}}{2 k_{B} T}+\frac{\mu}{k_{B} T}$, we finally obtain

$$
\mu<0
$$

This criterion is analogous to the nonrelativistic one and holds at all temperatures far from absolute zero, similar to the nonrelativistic case.

3) Considering $m_{c}$ as a parameter, in order to determine absolute temperature for thermal equilibrium, one should have, in accordance with (13), $2 A m_{c}>0$. It leads to $m_{c}>0$, since $A>0$, and, through (47), to

$$
\mu_{K} \geq 0
$$

The three conditions (72)-(74) constitute the set of criteria for validity of the theory discussed in the present paper.

The dependencies of $\mu$ and $\mu_{K}$ on $T$ are determined by equation (63) which in the low-temperature limit transforms to (67). The corresponding equation for the high-temperature case can be obtained from the normalization relation of relativistic statistical mechanics of distinguishable events discussed in [6],

$$
n=\frac{1}{(2 \pi)^{3}} \frac{M^{2}}{\mu_{K}^{2}}\left(k_{B} T\right)^{2} e^{\frac{\mu}{k_{B} T}} \Psi\left(2,2 ; \frac{M}{2 \mu_{K} k_{B} T}\right),
$$

by taking the limit $T \rightarrow \infty[6,(30)]$ :

$$
n=\frac{1}{4 \pi^{3}} \frac{M}{\mu_{K}}\left(k_{B} T\right)^{3} e^{\frac{\mu}{k_{B} T}} .
$$

Summarizing, we can write down the following relations, taking into account the three cases considered above,

$$
n= \begin{cases} \pm \frac{1}{2 \pi^{3}}\left(k_{B} T\right)^{4} L_{4}\left( \pm e^{\frac{\mu}{k_{B} T}}\right), & \text { low temperatures } \\ \frac{1}{(2 \pi)^{3}} \frac{M^{2}}{\mu_{K}^{2}}\left(k_{B} T\right)^{2} \sum_{s=1}^{\infty}\left\{( \pm 1)^{s+1} e^{\frac{s \mu}{k_{B} T}} \Psi\left(2,2 ; \frac{s M}{2 \mu_{K} k_{B} T}\right) / s^{2}\right\}, & \text { intermediate case } \\ \frac{1}{4 \pi^{3}} \frac{M}{\mu_{K}}\left(k_{B} T\right)^{3} e^{\frac{\mu}{k_{B} T}} . & \text { high temperatures }\end{cases}
$$

\section{Concluding remarks}

We have considered an equilibrium relativistic ensemble of indistinguishable events, described by an equilibrium relativistic distribution function representing covariant generalization of the known nonrelativistic Bose-Einstein/Fermi-Dirac distributions, 
with variable mass. We have shown that this distribution can be identified with a grand canonical distribution function first introduced by Horwitz, Schieve and Piron in their work on static Gibbs ensembles.

We have found that an equilibrium state of such a system is characterized by a well-defined mass distribution, following directly from the equilibrium relativistic distribution, by integration over angular and hyprebolic angular variables. The normalization relation of this distribution represents an equation linking the basic thermodynamic properties of the system $\mu, \mu_{K}, n, T$.

The dependence of $\mu$ on $T$ is explicitly defined only for low temperatures. Although the dependence of $\mu_{K}$ on $T$ is not determined, several remarks on this point can be made. Miller and Suhonen [16], studying possible applications of grand canonical distribution function of ref. [11] in high-energy physics, showed that at low temperatures one should have $\mu_{K}<<1$, whenever at high temperatures $\mu_{K}>>1$. The former relation may also justify the fact that it is possible to omit the term $\frac{\mu_{K} m^{2}}{2 M}$ in comparison with $(E-\mu)$ in the exponent of the equilibrium relativistic distribution function in the low-temperature limit.

Finally, we have shown that in the Galilean limit a statistical mechanics of indistinguishable events discussed in the present paper passes over to the usual nonrelativistic statistical mechanics of indistinguishable particles. 


\section{References}

[1] L. Burakovsky and L.P. Horwitz, Tel-Aviv Univ. Preprint TAUP-2092-93

[2] L.P. Horwitz and C. Piron, Helv. Phys. Acta 46 (1973) 316

[3] R. Arshansky, L.P. Horwitz and Y. Lavie, Found. Phys. 13 (1983) 1167

[4] J.D. Jackson, Classical Electrodynamics, 2nd ed., (Wiley, New-York, 1975)

[5] L.P. Horwitz, S. Shashoua and W.C. Schieve, Physica A 161 (1989) 300

[6] L. Burakovsky and L.P. Horwitz, Physica A, in press

[7] L. Burakovsky and L.P. Horwitz, Tel-Aviv Univ. Preprint TAUP-2081-93

[8] J.L. Synge, The Relativistic Gas, (North-Holland, Amsterdam, 1957)

[9] I.S. Gradshteyn and I.M. Ryzhik, Tables of Integrals, Series, and Products, (Academic Press, New York, 1980) p.958, subsection 8.432, formula 2; p.717, subsection 6.631, formula 3; Ref.[10], p.264, section 6.9, formula (2).

[10] Higher Transcendental Functions, Bateman Series, Ed. A.Erdélyi, Vol.1, p.278, subsection 6.13.1, (McGraw-Hill, New-York, 1953)

[11] L.P. Horwitz, W.C. Schieve and C. Piron, Ann. Phys. (N.Y.) 137 (1981) 306

[12] L.P. Horwitz and F.C. Rotbart, Phys. Rev. D 24 (1981) 2127

[13] L. Burakovsky and L.P. Horwitz, in preparation

[14] M. Abramowitz and I.A. Stegun, Handbook of Mathematical Functions, p.375, (Dover, New-York, 1970)

[15] A.P. Prudnikov et al. Integrals and series, (Gordon and Breach, New York, 1980) Vol. 3, p.762, Appendix II.5

[16] D.E. Miller and E. Suhonen, Phys. Rev. D 26 (1982) 2944 